\title{
Cold snare polypectomy effectively reduces polyp burden in familial adenomatous polyposis
}

Authors

Institution
Nedhi J. Patel, Prasanna L. Ponugoti, Douglas K. Rex

Department of Medicine, Division of Gastroenterology/Hepatology, Indiana University School of Medicine, Indianapolis, IN, USA submitted

11. November 2015

accepted after revision

12. February 2016

\section{Bibliography}

DOI http://dx.doi.org/

10.1055/s-0042-104114

Published online: 30.3.2016

Endoscopy International Open

2016; 04: E472-E474

(c) Georg Thieme Verlag KG

Stuttgart · New York

E-ISSN 2196-9736

Corresponding author

Douglas K. Rex, MD

550 N. University Hospital Blvd. Suite 4100

Indianapolis

IN 46202

USA

Fax: +1-317-944-5449

drex@iu.edu
Background and study aims: Familial adenomatous polyposis (FAP) is generally managed by colectomy, but in some cases surgery is delayed and polyp burdens are managed endoscopically. We aimed to describe the use of cold snare polypectomy to control the polyp burden in selected patients with FAP.

Patients and methods: This was a retrospective cohort study. Polyps were counted and the range of polyp size recorded at each examination. Patients with a reduction in polyp number and mean size were considered to have successful endoscopic reduction of their polyp burdens.

Results: Of 79 patients with FAP, 21 had an attempt at delaying surgery by cold snaring of at

\section{Introduction}

$\nabla$

Familial adenomatous polyposis (FAP) accounts for less than $1 \%$ of all colorectal cancers [1]. The traditional treatment is total proctocolectomy, though subtotal colectomy is used in some cases that are accompanied by low rectal polyp burdens. Patients with low polyp burdens may choose to delay colectomy to avoid complications of surgery, including the potential for development of desmoid tumor, loss of fecundity in women, and diarrhea after total proctocolectomy. For patients who have undergone total proctocolectomy, endoscopic control of a high polyp burden is important to avoid any consideration of surgical resection of the ileo-anal pouch.

In this report, we described the use of large-scale cold snare polypectomy to delay surgery in FAP patients.

\section{Materials and methods}

Procedures were performed with Olympus (Olympus Corporation, Center Valley, PA, United States) colonoscopes or upper endoscopes and least 30 adenomas, and had at least one followup at our institution. Ten patients had intact colons, 6 had intact rectums, and 5 had heavy polyp burdens in an ileo-anal pouch. Among the 21 patients, the mean number of polyps resected at the first examination was 85 , range $30-342$. Nineteen of 21 patients had fewer polyps at the second examination, and of those, only one had any persistence of adenomas $\geq 1 \mathrm{~cm}$ in size. During follow-up, two patients underwent surgical resection and the remainder had reductions in their polyp burdens at follow-up endoscopy.

Conclusions: Cold snare polypectomy effectively reduces polyp burden in selected FAP patients.

cold snaring was performed with an 11-mm Captiflex (Boston Scientific, Marlborough, MA, United States) snare.

We retrospectively examined a database of patients with polyposis syndromes managed by one of the authors (DKR) and maintained at Indiana University Hospital and identified patients with FAP. Patients with FAP were excluded if they had undergone total proctocolectomy and did not have significant polyp burden in the ileo-anal pouch, or if they had an intact colon or rectum and had never had more than 30 polyps removed in one procedure in an attempt to endoscopically control polyp burden, if they had an intact colon or rectum and had a polyp burden that was too great for attempted endoscopic control, or if they had one procedure and no follow-up at our center. All of the patients were referred to DKR. Permission to review the de-identified database was granted by the Institutional Review Board at Indiana University Health on June 15, 2015.

All procedures were performed at Indiana University Hospital between July 2004 and January 2016. All procedures were performed with standard Olympus high resolution or high definition colonoscopes without chromoendoscopy. The ini- 


\begin{tabular}{|lclll|}
\hline Patient anatomy & \multicolumn{2}{c|}{ Number of polyps removed at initial procedure } & \multicolumn{2}{c|}{ Initial procedure time, min } \\
\cline { 2 - 5 } & Mean & Range & Mean & Range \\
\hline Intact colon & 78 & $30-200$ & 49 & $19-85$ \\
\hline Intact rectum & 78.5 & $30-155$ & 45 & $24-56$ \\
\hline Ileo-anal pouch & 110.6 & $30-342$ & 62 & $28-96$ \\
\hline
\end{tabular}

Table 1 Number of polyps removed and procedure times at the initial resection by patient anatomy. tial and subsequent procedures and polypectomies were considered successful if the number of polyps detected at follow-up examinations was similar to or lower than previous examinations and if no or only a few large polyps ( $\geq 1 \mathrm{~cm}$ in size) were detected at follow-up examinations. In the initial endoscopic procedure performed to control polyp burden, polyps $\geq 1 \mathrm{~cm}$ in size were removed by hot snaring, including endoscopic mucosal resection in some cases. Polyps $<1 \mathrm{~cm}$ in size were removed by cold snaring. A nurse counted the number of polyps cold snare resected as they were removed. In patients with intact colons, polyps were typically resected from the cecum and ascending colon with no attempt at retrieval until resections were completed in those sections of the colon. Once the cecum and the ascending colon were cleared, the snare was withdrawn and the detected polyps were suctioned into a trap for pathologic examination. That process was then repeated for the transverse colon, the left colon including the descending and sigmoid colon, and finally the rectum. The range of sizes of polyps was recorded for each section of the bowel.

Complications were identified by chart review, direct interview at follow-up examination, and follow-up telephone call 24-72 hours after the procedure.

No statistical analysis was performed as the results are descriptive.

\section{Results}

There were 79 patients with FAP in the database. There were 21 (11 female) patients with an intact colon or rectum or ileo-anal pouch, in which an initial examination included at least 30 polyps removed by cold snare polypectomy, and in which the patient had at least one follow-up at our institution. All of the patients apart from one had known adenomatous polyposis coli (APC) mutations or were family members of patients with known APC mutations. The mean age of the 21 patients was 37 years, and 7 of the 10 patients with intact colons were under age 30. Of these 21 patients, 10 had intact colons at the first examination, 6 had intact rectums, and 5 were status post total colectomy with significant polyp burdens in the ileo-anal pouch. All of the patients were referred to DKR by other gastroenterologists. Only one of the pouch patients was being considered for surgery by the referring physician, and this patient had a very heavy pouch polyp burden (see below). Across the 21 patients, more than $99 \%$ of all polyp resections were performed by cold snare polypectomy. There were seven patients who received nonsteroidal anti-inflammatory drugs (NSAIDs) throughout the study interval, one received aspirin, and the remainder were not on NSAIDs. All but two had been placed on NSAIDs before referral to our center and did not have an apparent response.

The number of procedures performed to control the polyp burden ranged from 2 to 5, with mean 3.1. Mean follow-up was 10.7 months.
There were two patients referred for surgical resection during follow-up. One of these had an intact colon and had 57 polyps removed at the baseline colonoscopy and 105 at a second procedure 13 months later, though no polyps $>1 \mathrm{~cm}$ in size were identified at the follow-up.This patient was then referred for total proctocolectomy. A second patient with an intact colon had 200 polyps removed in a first colonoscopy, 20 in a second colonoscopy performed 10 months later, and 50 at a third colonoscopy 11 months after the second. In a fourth colonoscopy, 60 were removed from the rectum only, and the patient was then referred for subtotal colectomy.

For all patients, the mean number of polyps removed in the first examination was 85 , median 65, range 30-342. For all 21 patients, the number of polyps removed at the second procedure was mean 40 , median 31, range 2-105. Other than the two patients referred to surgery, polyp counts in all patients went down at subsequent examinations. For example, the largest number of polyps resected in one procedure was 342 in a patient with an ileo-anal pouch who had neglected follow-up for 7 years. At the second procedure performed 3 months later, 28 polyps were removed by cold snare. This was the only patient who still had polyps $\geq 10 \mathrm{~mm}$ at the second procedure. No patient developed colorectal cancer or cancer in the pouch during follow-up. No patient had a complication from polypectomy. All polyps resected were tubular or tubulovillous adenomas and no adenoma $<10 \mathrm{~mm}$ in size had high grade dysplasia.

- Table 1 shows the number of polyps removed at the initial procedure and the procedure times for the initial procedure grouped by patient anatomy. For example, the longest initial procedure was 96 minutes in the pouch patient who had 342 polyps removed during that procedure, or an average of 3.6 polyps per minute for the entire procedure.

\section{Discussion}

$\nabla$

In this report, we describe reduction of polyp burdens in retained colon, rectum or ileo-anal pouch in a subset of patients with FAP in whom at least 30 lesions were removed and polypectomies were performed largely with cold snaring. In an initial endoscopic procedure, there was an attempt to achieve endoscopic control of polyp burdens, and at least one follow-up examination was performed at our institution. No patient developed cancer and only one had any large adenomas during follow-up.

To our knowledge, no previous report has described the use of large scale cold snaring to control the polyp burden in FAP. Reliance on large scale cold snaring rather than ablative tools such as argon plasma coagulation (APC) is the unique and novel aspect of this report. Cold snaring has the potential to be more effective than APC, since snaring is more effective than ablation [2], and less likely to produce complications since cold techniques produce fewer complications than other methods [3].

Limitations of this study include its retrospective nature and limited follow-up in some patients. However, all patients had at least 
one follow-up and all but two patients had fewer and smaller polyps at follow-up. In some of our patients, continued chemoprevention may have helped reduce polyp burdens [4,5]. The relative importance of cold snaring and chemoprevention in some patients was uncertain and endoscopic therapy and chemoprevention are often considered complimentary approaches to polyp control in FAP [6-9]. We used the approach of large scale cold snaring in about a quarter of our FAP patients. Thus, most patients with FAP in our database had either already undergone total proctocolectomy and were referred for management of duodenal lesions, or had subtotal colectomies and had a relatively low total burden of rectal polyps (some needed endoscopic mucosal resection of a large rectal polyp but did not have rectal carpeting). The patients described in this series had polyps burdens that were larger but were subjectively endoscopically manageable $[10,11]$, typically wanted to delay surgery (e.g. until after childbearing, because of a family history of desmoids, or to maintain quality of life by retaining a rectum or ileo-anal pouch in a patient who had already been operated), and indicated they were ready to adhere to recommended follow-up. Assessing what is an endoscopically manageable polyp burden is partly subjective $[10,11]$, but includes consideration of the number and size of polyps and whether colectomy has already been performed. The patient with 342 polyps resected in the first examination was considered a candidate for endoscopic management because of the importance of preserving his ileo-anal pouch. Clearly, the polyp size range appropriate for endoscopic management is considerably larger in a patient who has already had a total or abdominal colectomy compared to the patient with an intact colon [1]. Although we describe here the use of cold snaring in patients with substantial polyp burdens (at least 30 polyps), to demonstrate that cold snaring can reduce polyp numbers in FAP patients, anecdotally we also commonly use cold snaring in the rectum or pouch of FAP patients with low polyp burdens at surveillance examinations.

Ablation using APC is commonly used in clinical practice to reduce polyp burdens in patients with FAP after subtotal colectomy or total proctocolectomy. APC is efficient and safe, and the senior author has extensive experience in its use in FAP patients for colonic, ileal, and duodenal polyps. Anecdotally, we find that cold snaring is as efficient as APC in colorectal polyp obliteration in FAP patients, particularly for polyps in the $4-10 \mathrm{~mm}$ size range. Such polyps often require multiple aimed short pulses of APC, or a longer sustained pulse of APC, but are eradicated in a single quick cut using the cold snare. Eradication of very flat $1-3 \mathrm{~mm}$ polyps with cold snaring seems sometimes to be more efficient with APC. Use of a stiff snare that improves tissue capture may improve the efficiency of cold snaring for tiny flat polyps. Thus, we find that cold snaring has generally comparable efficiency to APC in treating colorectal polyps in FAP, and suspect it is at least as safe and effective as APC.

Attempts to delay surgery in FAP may be appropriately met with skepticism. In a study of 659 FAP patients with subtotal colectomies, the risk of death from rectal cancer was $12.5 \%$ by age 65 , and $75 \%$ of patients with rectal cancer had a negative rectoscopy within 12 months of the diagnosis of rectal cancer [9]. This report is inconsistent with our experience (with fewer patients) in that we have never seen a patient with FAP progress to colorectal or pouch cancer during surveillance, and we rarely see a "negative" proctoscopy during surveillance of FAP after subtotal colectomies. In almost all cases, at least a few diminutive polyps are en- countered, and we resect or destroy these polyps. Our experience is more consistent with the report of Gleeson et al. where only three of 42 FAP patients with prior surgical treatment had neoplastic progression during endoscopic surveillance. In that series, polyp destruction was achieved using a combination of snare polypectomy, APC, and Nd:YAG laser [8]. We recommend that endoscopic examination during FAP surveillance should be meticulous, should include large scale polyp resection or destruction, and that the number of treated polyps be counted (and the size range recorded) so that reduction of polyp can be documented at subsequent examination.

Our results indicate that large scale cold snare polypectomy provides an effective approach to controlling polyp burden in selected FAP patients, and can be considered a viable alternative to ablative techniques such as APC. Because cold snare polypectomy is efficient (and safe) in its application, we recommend it as an approach to controlling polyp burden in selected individuals with FAP.

Competing interests: Yes. Olympus: Research Support, Consulting Fees.

\section{Acknowledgments}

$\nabla$

This work was funded by a gift to the Indiana University Foundation in the name of Douglas K. Rex from Scott and Kay Schurz of Bloomington, Indiana, and their children.

\section{References}

1 Young Y, Terdiman JP. Endoscopic management of familial colonic neoplasia. Gastrointest Endosc Clin North Am 2005; 15: 549-580

2 Moss A, Williams SJ, Hourigan LF et al. Long-term adenoma recurrence following wide-field endoscopic mucosal resection (WF-EMR) for advanced colonic mucosal neoplasia is infrequent: results and risk factors in 1000 cases from the Australian Colonic EMR (ACE) study. Gut 2015; 64: 57-65

3 Horiuchi A, Nakayama Y, Kajiyama $M$ et al. Removal of small colorectal polyps in anticoagulated patients: a prospective randomized comparison of cold snare and conventional polypectomy. Gastrointest Endosc 2014; 79: 417-423

4 Giardiello FM, Hamilton SR, Krush AJ et al. Treatment of colonic and rectal adenomas with sulindac in familial adenomatous polyposis. NEJM 1993; 328: $1313-1316$

5 Steinbach G, Lynch PM, Phillips RK et al. The effect of celecoxib, a cyclooxygenase-2 inhibitor, in familial adenomatous polyposis. NEJM 2000; 342: 1946-1952

6 West NJ, Clark SK, Phillips RK et al. Eicosapentaenoic acid reduces rectal polyp number and size in familial adenomatous polyposis. Gut 2010; 59: $918-925$

7 Cruz-Correa M, Shoskes DA, Sanchez P et al. Combination treatment with curcumin and quercetin of adenomas in familial adenomatous polyposis. Clin Gastroenterol Hepatol 2006; 4: 1035-1038

8 Gleeson FC, Papachristou GI, Riegert-Johnson DL et al. Progression to advanced neoplasia is infrequent in post colectomy familial adenomatous polyposis patients under endoscopic surveillance. Fam Cancer 2009; 8: $33-38$

9 Vasen HF, van Duijvendijk P, Buskens E et al. Decision analysis in the surgical treatment of patients with familial adenomatous polyposis: a Dutch-Scandinavian collaborative study including 659 patients. Gut 2001; 49: $231-235$

10 Debinski HS, Love S, Spigelman AD et al. Colorectal polyp counts and cancer risk in familial adenomatous polyposis. Gastroenterology 1996; 110: $1028-1030$

11 Lynch PM, Morris JS, Ross WA et al. Global quantitative assessment of the colorectal polyp burden in familial adenomatous polyposis by using a web-based tool. Gastrointest Endosc 2013; 77: 455 - 463 